\title{
Quantized contact angles in the dewetting of a structured liquid
}

Article

Published Version

Ilton, M., Stasiak, P., Matsen, M. W. and Dalnoki-Veress, K. (2014) Quantized contact angles in the dewetting of a structured liquid. Physical Review Letters, 112 (6). 068303. ISSN 0031-9007 doi:

https://doi.org/10.1103/PhysRevLett.112.068303 Available at https://centaur.reading.ac.uk/67530/

It is advisable to refer to the publisher's version if you intend to cite from the work. See Guidance on citing.

Published version at: http://link.aps.org/doi/10.1103/PhysRevLett.112.068303

To link to this article DOI: http://dx.doi.org/10.1103/PhysRevLett.112.068303

Publisher: American Physical Society

All outputs in CentAUR are protected by Intellectual Property Rights law, including copyright law. Copyright and IPR is retained by the creators or other copyright holders. Terms and conditions for use of this material are defined in the End User Agreement.

\section{www.reading.ac.uk/centaur}

\section{CentAUR}

Central Archive at the University of Reading 
Reading's research outputs online 


\title{
Quantized Contact Angles in the Dewetting of a Structured Liquid
}

\author{
Mark Ilton, ${ }^{1}$ Pawel Stasiak, ${ }^{2}$ Mark W. Matsen, ${ }^{2,3}$ and Kari Dalnoki-Veress ${ }^{1,4, *}$ \\ ${ }^{1}$ Department of Physics and Astronomy and the Brockhouse Institute for Materials Research, \\ McMaster University, Hamilton, Ontario L8S 4M1, Canada \\ ${ }^{2}$ School of Mathematical and Physical Sciences, University of Reading, Whiteknights, Reading RG6 6AX, United Kingdom \\ ${ }^{3}$ Waterloo Institute for Nanotechnology, University of Waterloo, Waterloo, Ontario N2L 3G1, Canada \\ ${ }^{4}$ Laboratoire de Physico-Chimie Théorique, UMR CNRS Gulliver 7083, ESPCI, Paris, France
}

(Received 3 October 2013; revised manuscript received 10 December 2013; published 14 February 2014)

\begin{abstract}
We investigate the dewetting of a disordered melt of diblock copolymer from an ordered residual wetting layer. In contrast to simple liquids where the wetting layer has a fixed thickness and the droplets exhibit a single unique contact angle with the substrate, we find that structured liquids of diblock copolymer exhibit a discrete series of wetting layer thicknesses each producing a different contact angle. These quantized contact angles arise because the substrate and air surfaces each induce a gradient of lamellar order in the wetting layer. The interaction between the two surface profiles creates an effective interface potential that oscillates with film thickness, thus, producing a sequence of local minimums. The wetting layer thicknesses and corresponding contact angles are a direct measure of the positions and depths of these minimums. Self-consistent field theory is shown to provide qualitative agreement with the experiment.
\end{abstract}

DOI: 10.1103/PhysRevLett.112.068303

PACS numbers: 82.35.Jk, 68.08.Bc, 68.15.+e

The dewetting of a simple liquid from a solid substrate is a familiar process, such as when water droplets form on the waxy surface of an automobile. Small droplets, where gravity can be ignored, form spherical caps (see schematic in Fig. 1) with a contact angle, $\theta$, given by

$$
\Phi\left(h_{\min }\right)=\gamma(\cos \theta-1),
$$

where $\gamma$ is the interfacial tension of the liquid-air surface and $\Phi(h) \equiv[F(h)-F(\infty)] / A$ is the effective interface potential between the two surfaces of the liquid film [1-3]. The latter is defined in terms of the free energy, $F(h)$, of a film with uniform thickness $h$ and area $A$. In general, dewetting leaves behind a microscopically thin residual wetting layer of thickness $h_{\min }$, corresponding to the minimum in $\Phi(h)$ [3]. For simple liquids such as water, $\Phi(h)$ has a single minimum as illustrated by the dashed curve in Fig. 1, which leads to droplets with a single contact angle. The behavior becomes more interesting for complex liquids such as liquid crystals in their isotropic (disordered) state, where surface-induced smectic (lamellar) order fades towards the center of the film. This should produce a series of minimums in $\Phi(h)$ with decaying depths, resulting in a discrete spectrum of contact angles. Although dewetting is a well-studied phenomenon in physics, these quantized contact angles have never been observed despite some experiments that come tantalizingly close to revealing such behavior [4-7]. Experiments on liquid crystalline polymers have shown a stepwise thinning of the wetting layer [4], a process which occurs reversibly in films of $n$ alkanes [7]. A partial reconstruction of $\Phi(h)$ around its global minimum was performed based on measurements of low molecular weight lubricant oils [5] as well as lamellar-forming diblock copolymers [6]. However, these studies did not observe discrete changes in the contact angle, though theoretically expected. Here, we examine the wetting behavior of a disordered melt of diblock copolymer on a silicon substrate. In this system, the different wetting layers are highly metastable ( $\sim$ days), which allows us to accurately resolve the quantized set of equilibrium contact

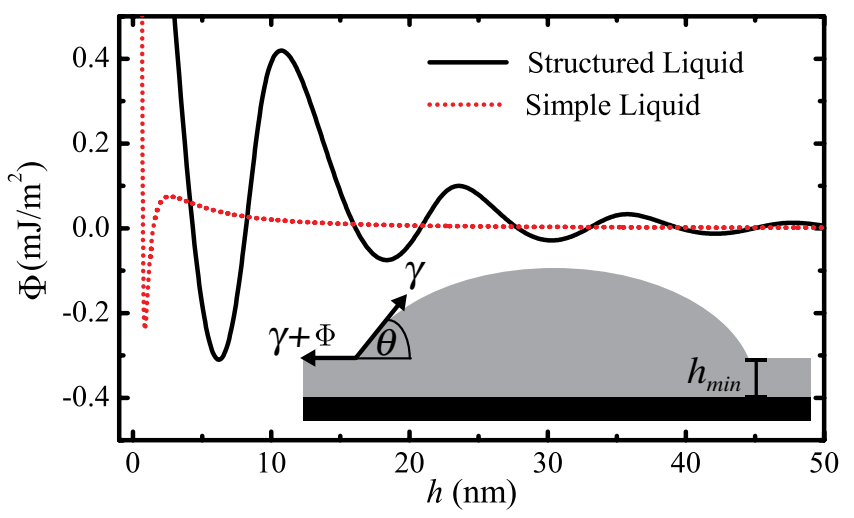

FIG. 1 (color online). Effective interface potential between the surfaces of a film as a function of the film thickness for simple and structured liquids. The inset shows the geometry of our experiment: partial dewetting of a film results in a thin wetting layer with $h=h_{\min }$ corresponding to a minimum in $\Phi(h)$ and a droplet with contact angle $\theta$ given by Eq. (1). The dashed curve is for polystyrene on an oxidized silicon substrate [2], and the solid line is calculated for diblock copolymer as described in the text. 
angles. Furthermore, self-consistent field theory (SCFT) calculations for structured polymers [8] exhibit qualitative agreement with the experimental observations.

A diblock copolymer is a linear polymer with two blocks of chemically distinct segments, typically labeled $A$ and $B$ [9]; the polymerization index (total number of segments) is denoted by $N=N_{A}+N_{B}$ and the composition is specified by $f=N_{A} / N$. The interaction between the segments is characterized by a dimensionless Flory-Huggins interaction parameter $\chi$, which varies inversely with temperature $T$. For most $A-B$ combinations, $\chi>0$, which implies a tendency for the unlike components to segregate into $A$ - and $B$-rich domains. This tendency depends on the product of the interaction strength and the size of the copolymer chains, $\chi N$. When $\chi N \gtrsim 10$ (corresponding to low temperatures), the domains form ordered periodic morphologies with different geometries depending on $f$. For a symmetric composition $(f=0.5)$, the diblocks self-assemble into thin flat $A B$ monolayers of alternating orientations, which creates a lamellar phase with a period equal to the $A B / B A$ bilayer thickness, $L_{0}$. As the temperature increases ( $\chi$ decreases) beyond the order-disorder transition (ODT) temperature, $T_{\mathrm{ODT}}$, thermal fluctuations destroy the longrange order producing a disordered phase.

In the presence of a flat surface, the domains align to form lamellae parallel to the surface provided that the surface has a sufficient affinity for one of the two components. This can happen even when $T>T_{\mathrm{ODT}}$, although the order of the lamellar layers progressively deteriorates with increasing distance from the surface, resulting in an oscillating concentration profile of period $L_{0}$ [10] that decays in amplitude [11-14]. In a thin film, the concentration profiles from opposing surfaces can interfere with each other causing the free energy, $F(h)$, to oscillate with a period of $L_{0}$. The preferred thicknesses occur when $h=\nu L_{0} / 2$, where $\nu$ is an even integer if the two surfaces have an affinity for the same component (symmetric wetting) and $\nu$ is odd if they have affinities for opposite components (asymmetric wetting). The solid curve in Fig. 1 shows the effective interface potential, $\Phi(h) \equiv[F(h)-F(\infty)] / A$, between two surfaces with opposite affinities, where the minimums occur for $\nu=1,3,5, \ldots$. Since the effect of the surfaces diminishes towards the bulk of the film, the minimums become shallower as the film thickness increases. Consequently, the diblock copolymer films tend to dewet one $A B / B A$ bilayer at a time $(\nu \rightarrow \nu-2)$, which should, theoretically, cause discrete jumps in the contact angle, given by Eq. (1), until the minimum thickness $(\nu=1)$ is reached. Although the thicker wetting layers $(\nu>1)$ are only metastable, the barriers between them can be relatively large, and thus, their lifetimes can be exceptionally long ( $\sim$ days).

In our experiments, the polystyrene-poly(2-vinylpyridine) (PS-P2VP) diblock copolymer had a total number-averaged
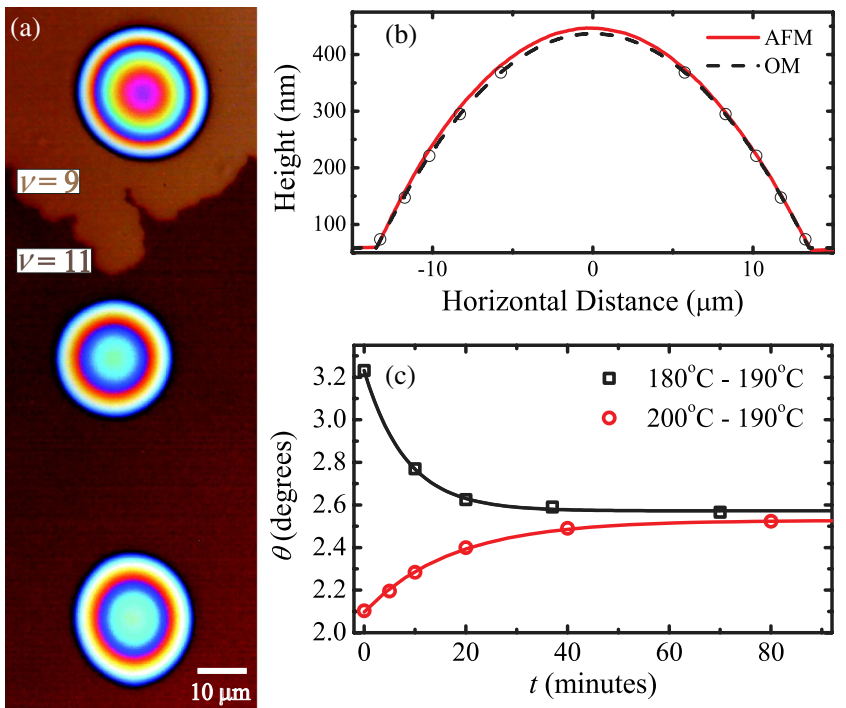

FIG. 2 (color online). (a) Color OM image of PS-P2VP diblock copolymer droplets coexisting with wetting layers of $\nu=9$ (light brown) and $\nu=11$ (dark brown). Differences in their contact angles are evident by the spacing of the optical interference rings. (b) Profile of the droplet with the $\nu=9$ wetting layer measured by AFM (solid red curve) and OM (open circles denote thicknesses obtained from the interference fringes, and the dashed curve is a spherical fit). The heights from AFM and OM agree to within $10 \mathrm{~nm}$, and the resulting contact angles are indistinguishable. (c) Measurements demonstrating that advancing (black squares) and receding (red circles) contact angles converge to within $0.1^{\circ}$ in about an hour.

molecular weight of $M_{n}=16.5 \mathrm{~kg} / \mathrm{mol}(N=158)$, a polydispersity index of 1.09 , and an equal composition of PS and P2VP (Polymer Source Inc., Canada). From previous studies, we know that $\chi=(97 \mathrm{~K}) / T-0.11$ [15] and $T_{\mathrm{ODT}} \approx 160^{\circ} \mathrm{C}$ [14]. Thin films were prepared by spin coating from dilute toluene solutions onto silicon substrates using slow spin-coating speeds (500-1000 rpm) in order to intentionally prepare nonuniform films. The gradients in the surface topography caused instabilities in the film when heated above $T_{\mathrm{ODT}}$ in an inert nitrogen environment (see movie in Supplemental Material [16]). The instabilities lead to dewetting with metastable wetting layers of different thicknesses as shown in Fig. 2(a).

Contact angle measurements were performed with atomic force microscopy (AFM, Veeco, Caliber) and with optical microscopy (OM, Olympus, USA) using a monochromatic filter (460 $\mathrm{nm}$ wavelength) to create interference fringes from which the profile of the droplet was inferred $[17,18]$. AFM and OM yield the same profile to within a few percent resulting in nearly identical values for the contact angle [see Fig. 2(b)]. Consistent with previous measurements [17], the AFM error signal shows steps near the contact line, indicating a small amount of residual order at the base of the droplets. Since the OM measurements are more easily performed, results were predominantly 
obtained using this technique. Both advancing and receding contact angles were measured and were in agreement to within $0.1^{\circ}$ [see Fig. 2(c)], indicating that our measurements are of the equilibrium contact angle and free from hysteresis or pinning effects. The metastable wetting layers were generally stable for several days, whereas the typical relaxation time for a droplet to reach its equilibrium contact angle is on the order of an hour [see Fig. 2(c)].

Dewetting was observed for temperatures ranging from $190^{\circ} \mathrm{C}$ to $240^{\circ} \mathrm{C}$, well above $T_{\mathrm{ODT}} \approx 160^{\circ} \mathrm{C}$, and for wetting layer thicknesses from $\nu=1$ to 15 ( $h \approx 7$ to $96 \mathrm{~nm}$ ). The contact angles ranged from $0.5^{\circ}$ (the lower limit of our measurement technique) for the thickest wetting layer to $8.5^{\circ}$ for the stable $\nu=1$ wetting layer. As shown in Fig. 3(a), there is a spectrum of discrete contact angles indicating multiple minimums in the effective interface potential [Eq. (1)] corresponding to different values of $\nu$. As the temperature is changed, the energy spectrum shifts. For thicker wetting layers, the contact angle decreases as a function of increasing temperature, while a more complex dependence is found for thinner wetting layers.

In order to develop a more complete understanding of the results, we calculate the free energy, $F(h)$, of a uniform film of symmetric diblock copolymer as a function of film thickness, $h$, using the standard SCFT for incompressible Gaussian chains [8]. Note that our calculations assume conformational symmetry, where the $A$ and $B$ segments occupy the same volume, $\rho_{0}^{-1}$, and have equal statistical segment lengths, $a$. Different values of temperature in the disordered phase, $T>T_{\mathrm{ODT}}$, are produced by varying $\chi N<10.495$. The substrate and air surfaces were treated by imposing reflecting boundaries with delta-function potentials acting on the two polymer components, as described previously $[19,20]$. The affinity of the substrate for $A$-type segments was controlled by the dimensionless parameter, $\Lambda_{\text {sub }}=\left(\gamma_{\text {sub } / A}-\gamma_{\mathrm{sub} / B}\right) / 2 k_{B} T \rho_{0} a N^{1 / 2}$, where

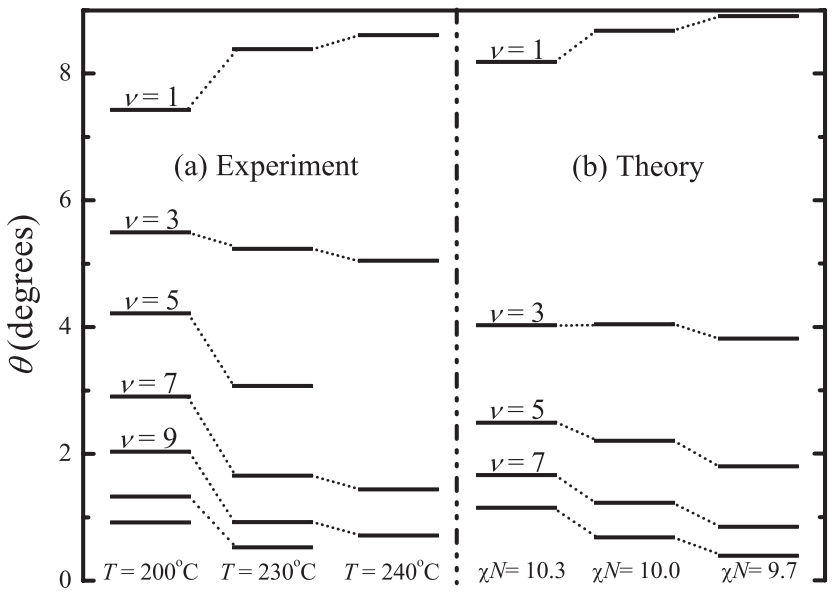

FIG. 3. The spectrum of quantized contact angles $\theta$, shown for different numbers of diblock monolayers, $\nu$, in the wetting layer. (a) Experimental and (b) theoretical results are presented for several different temperatures and values of $\chi N$, respectively. $\gamma_{\mathrm{sub} / \alpha}$ is the surface tension between the substrate and the $\alpha=A$ or $B$ polymer component [19,21]. Likewise, an analogous parameter, $\Lambda_{\text {air }}$, was defined for the air surface, which we chose with an opposite sign so as to reproduce the asymmetric wetting conditions of the experiment. To match the strong surface affinities characteristic of the experiment, the dimensionless amplitudes were chosen large enough $\left(\Lambda_{\text {sub }} N=-0.3\right.$ and $\left.\Lambda_{\text {air }} N=0.3\right)$ such that the concentration of $A$ and $B$ segments at the substrate and air surfaces, respectively, were relatively pure. Increasing the affinities beyond these magnitudes had little effect on the contact angles.

The SCFT is performed in terms of reduced parameters such as $h / a N^{1 / 2}$ and $F(h) / n k_{B} T$, where $n$ is the total number of molecules in the film. In order to explicitly evaluate $\Phi(h) \equiv[F(h)-F(\infty)] / A$ as a function of $h$, we estimated the volume occupied by a single diblock copolymer to be $N / \rho_{0} \approx 26 \mathrm{~nm}^{3}$ and the average end-to-end of an unperturbed diblock to be $a N^{1 / 2} \approx 8.9 \mathrm{~nm}$ [22]. The solid curve in Fig. 1 shows $\Phi(h)$ calculated for $\chi N=10.3$. As expected, $\Phi(h)$ exhibits a series of minimums with decaying depths corresponding to odd-integer values of $\nu$. Using Eq. (1) with an average air/polymer surface tension of $\gamma \equiv\left(\gamma_{\mathrm{air} / A}+\gamma_{\mathrm{air} / B}\right) / 2 \approx 31 \mathrm{~mJ} / \mathrm{m}^{2}$ [23], we can then extract a contact angle, $\theta$, for each of the free energy minimums.

In Fig. 3(b), the theoretical spectrum for $\theta$ is shown for three different values of $\chi N$, and compared to the experimental results in Fig. 3(a). The experimental and theoretical results all show a monotonic decrease with $\nu$. Furthermore, there is an increase in the difference between the contact angles for $\nu=1$ and $\nu=3$ as the system becomes more disordered, indicating a complex dependence of the contact angle on temperature. The temperature dependence of the interface potential inferred by the experiment [obtained by measuring $\theta$ and making use of Eq. (1)] is shown in Fig. 4(a). Here, the data for $\nu=1$ have been omitted because they exhibit a larger scatter, which we attribute to a partial ordering of the droplets close to the

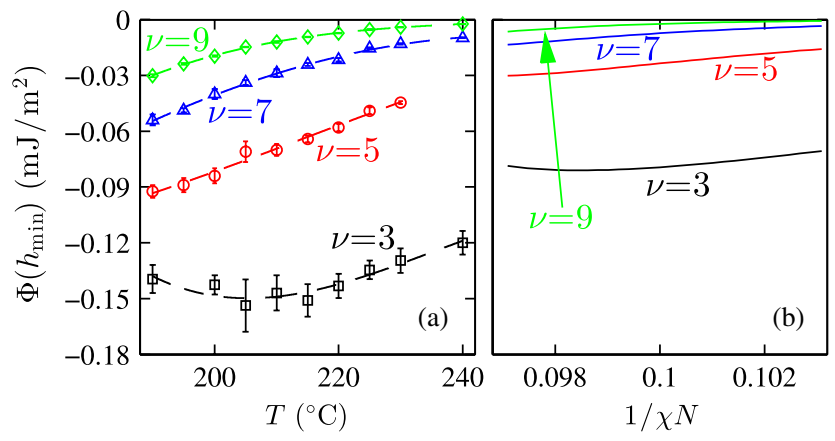

FIG. 4 (color online). The effective interface potential plotted as a function of (a) temperature for the experiments and (b) $1 / \chi N$ for theory. Results are shown for wetting layers with different numbers of diblock monolayers, $\nu$. Further results up to $\nu=15$ are provided in the Supplemental Material, Fig. S1 [16]. 
substrate as well as droplets pinning to small residual areas of $\nu=3$ where an extra bilayer has not fully dewetted (see Figs. S2-S3 in the Supplemental Material [16]). The corresponding results from SCFT calculations are plotted in Fig. 4(b), with the temperature dependence mimicked by $(\chi N)^{-1}$. The calculations are in good qualitative agreement with the experimental data. For $\nu \geq 5$, the contact angle decreases monotonically as a function of $T$ for the experiment or $(\chi N)^{-1}$ for the theory. Interestingly, this dependence becomes nonmonotonic at $\nu=3$ in both theory and experiment. A complete data set from $\nu=1$ to 15 is presented in the Supplemental Material (see Fig. S1) [16]. In addition to our results for PS-P2VP diblocks, we have observed similar behavior for PS-PMMA diblocks (see Supplemental Material, Fig. S4 [16]) where the substrate has a strong affinity for PMMA while the air has only a slight affinity for PS, which illustrates the generality of our observations.

This behavior is best observed at temperatures just above the ODT. At higher temperatures, the metastable minimums in $\Phi(h)$ will be too short lived to observe their contact angles. However, it is also essential that the system remains above the ODT so that $\Phi(h) \rightarrow 0$ as $h \rightarrow \infty$, or else there will be no driving force for films to dewet. Indeed, droplets of ordered diblock copolymer are known to slowly spread [24], indicative of the fact that they do not possess an equilibrium contact angle.

The physics of this dewetting behavior is relatively simple. The affinities of the substrate and air surfaces favor the formation of ordered lamellae so as to minimize the surface energy, but entropy favors disorder given that $T>T_{\text {ODT }}$. So as to minimize the number of ordered layers in a thin film while still maintaining the order next to the two surfaces, polymer is transferred from the film to the droplet where it can exist in a disordered state of lower free energy. The increased preference for thinner wetting layers (i.e., smaller $h_{\min }$ ) causes a quantized reduction in the effective interface potential. This leads to a change in the force balance at the contact line of the droplets, driving flow of material from the edge of the droplets towards their centers and, thus, a monotonic increase in $\theta$ with decreasing $\nu$ (see Fig. 3). The complicated temperature dependence of $\theta$ (see Fig. 4), on the other hand, is difficult to explain because of the numerous competing effects, such as the free energy difference between the ordered and disordered phases, the surface energies, and the decay length of the surface ordering. Nevertheless, SCFT captures the qualitative features. We note that accurate quantitative predictions should not be expected from SCFT because the mean-field approximation ignores composition fluctuations, which become important in the disordered phase [25].

The wetting layer is generally very thin for simple liquids, whereas it can be more than an order of magnitude thicker for structured liquids (compare the locations of the minimums in Fig. 1 for the two cases). It is for this reason that researchers $[4,6,26]$ have referred to the latter case as autophobic dewetting, meaning that the droplet dewets from what is essentially itself. In true instances of autophobic dewetting, such as a homopolymer dewetting from a chemically identical polymer brush [27-34] or elastomer [35-37], the droplet actually sits on top of a polymeric substrate which is anchored. In contrast, the wetting layer here does not extend beneath the droplet but rather coexists side-by-side with the droplet. Although there are ordered layers at the base of the droplet, the number of layers and their detailed composition profile are distinct from those of the wetting layer. For instance, all three droplets in Fig. 2(a) would possess identical microstructure next to the substrate despite being in contact with two different wetting layers $(\nu=9$ and 11). The only effect of $\nu$, or rather the depth of the effective interface potential $\Phi\left(h_{\text {min }}\right)$, is to modify the amount of force pushing material into the droplet, which in turn alters the Laplace pressure inside the droplet and, thus, the curvature of the polymer-air surface.

In summary, we have discovered a series of quantized contact angles when a film of structured isotropic liquid dewets from a solid substrate, specifically a disordered melt of diblock copolymer from a silicon wafer. The behavior results from a subtle competition between surface interactions that tend to order the film and entropy which favors the disordered state. Because the induced order is unfavorable above the ODT temperature, the film dewets so as to produce droplets of disordered diblock copolymer. The wetting layer left behind remains relatively ordered, and consequently, it forms, in our case, an odd number of diblock monolayers $(\nu=1,3, \ldots, 15)$. Since the surfaceinduced order decays at temperatures above the ODT, the monolayers at the center of a thick wetting layer will be somewhat disordered, and therefore, the tendency to dewet is relatively weak, resulting in a small contact angle. However, as the wetting layer thins, it becomes more ordered throughout, and consequently, the contact angle increases. It is this increase in contact angle due to the improved order of thinner wetting layers coupled with the step changes in thickness that creates the discrete spectrum of contact angles. We were able to observe the different angles with remarkable accuracy due to the long lifetimes of the metastable wetting layers $(\nu>1)$. In fact, the lifetimes were sufficient for us to carefully probe temperature dependence. Given that the set of $\theta$ and $h_{\min }$ is such a direct and accurate measure of the free energy, these experiments will provide a superb test for future calculations beyond SCFT $[38,39]$. Furthermore, the physics of this new dewetting behavior is reasonably generic to structured liquids, and thus, we expect it to occur in numerous other systems beyond the two lamellar diblock copolymer examples used in this Letter.

Financial support for this work was provided by NSERC (Canada) and EPSRC (U.K.). 
*dalnoki@mcmaster.ca

[1] A. N. Frumkin, J. Phys. Chem. 12, 337 (1938).

[2] R. Seemann, S. Herminghaus, and K. Jacobs, Phys. Rev. Lett. 86, 5534 (2001).

[3] K. Jacobs, R. Seemann, and S. Herminghaus, in Polymer Thin Films, edited by O. K. C. Tsui and T. P. Russell (World Scientific, Singapore, 2008), Chap. 10, pp. 243-265.

[4] S. Sheiko, E. Lermann, and M. Möller, Langmuir 12, 4015 (1996).

[5] R. Waltman, A. Khurshudov, and G. Tyndall, Tribol. Lett. 12, 163 (2002).

[6] R. Limary, P. F. Green, and K. R. Shull, Eur. Phys. J. E 8, 103 (2002).

[7] P. Lazar, H. Schollmeyer, and H. Riegler, Phys. Rev. Lett. 94, 116101 (2005).

[8] M. W. Matsen, J. Phys. Condens. Matter 14, R21 (2002).

[9] F. S. Bates, Science 251, 898 (1991).

[10] Near an interface which has a chemical preference for one of the two blocks, the lamellar spacing increases slightly from its equilibrium bulk value $L_{0}$ and is a function of the distance from the interface.

[11] G. H. Fredrickson, Macromolecules 20, 2535 (1987).

[12] A. Menelle, T. P. Russell, S. H. Anastasiadis, S. K. Satija, and C. F. Majkrzak, Phys. Rev. Lett. 68, 67 (1992).

[13] P. Mansky, T. P. Russell, C. J. Hawker, J. Mays, D. C. Cook, and S. K. Satija, Phys. Rev. Lett. 79, 237 (1997).

[14] A. B. Croll, A.-C. Shi, and K. Dalnoki-Veress Phys. Rev. E 80, 051803 (2009).

[15] A. B. Croll, M. W. Matsen, A.-C. Shi, andK. DalnokiVeress, Eur. Phys. J. E 27, 407 (2008).

[16] See Supplemental Material at http://link.aps.org/ supplemental/10.1103/PhysRevLett.112.068303 for a time-lapsed video of the droplet instability and supplemental Figs. S1-S4.

[17] A. B. Croll and K. Dalnoki-Veress, Eur. Phys. J. E 29, 239 (2009).

[18] S. Cormier, J. McGraw, T. Salez, E. Raphaël, and K. Dalnoki-Veress, Phys. Rev. Lett. 109, 154501 (2012).

[19] M. W. Matsen, Macromolecules 39, 5512 (2006).
[20] We neglected the long-range van der Waals interaction in our SCFT calculations because for PS-P2VP diblock copolymers this interaction only contributes a small correction to the effective interface potential. Including the van der Waals interaction would result in $\mathrm{a}<1^{\circ}$ reduction in the contact angles for $\nu=1$; the effect becomes negligible for the thicker wetting layers $(\nu=3,5, \ldots)$.

[21] M. W. Matsen, J. Chem. Phys. 106, 7781 (1997).

[22] L. J. Fetters, D. J. Lohse, D. Richter, T. A. Witten, and A. Zirkel, Macromolecules 27, 4639 (1994).

[23] B. B. Sauer and G. T. Dee, Macromolecules 35, 7024 (2002).

[24] A. B. Croll, M. V. Massa, M. W. Matsen, and K. DalnokiVeress, Phys. Rev. Lett. 97, 204502 (2006).

[25] F. S. Bates, J. H. Rosedale, G. H. Fredrickson, and C. J. Glinka, Phys. Rev. Lett. 61, 2229 (1988).

[26] M. W. J. van der Wielen, M. A. Cohen Stuart, and G. J. Fleer, Langmuir 14, 7065 (1998).

[27] R. Yerushalmi-Rozen, J. Klein, and L. J. Fetters, Science 263, 793 (1994).

[28] G. Reiter, P. Auroy, and L. Auvray, Macromolecules 29, 2150 (1996).

[29] G. Reiter and R. Khanna, Phys. Rev. Lett. 85, 5599 (2000).

[30] G. Reiter and R. Khanna, Langmuir 16, 6351 (2000).

[31] A. Voronov and O. Shafranska, Langmuir 18, 4471 (2002).

[32] J. H. Maas, G. J. Fleer, F. A. M. Leermakers, and M. A. Cohen Stuart, Langmuir 18, 8871 (2002).

[33] G. Reiter, Macromol. Symp. 229, 81 (2005).

[34] W. Béziel, G. Reiter, E. Drockenmuller, R.-V. Ostaci, S. Al Akhrass, F. Cousin, and M. Sferrazza, Europhys. Lett. 90, 26008 (2010).

[35] T. Kerle and J. Klein, Europhys. Lett. 38, 207 (1997).

[36] T. Kerle, R. Yerushalmi-Rozen, and J. Klein, Macromolecules 31, 422 (1998).

[37] J. Jopp and R. Yerushalmi-Rozen, Macromolecules 32, 7269 (1999).

[38] J. Qin and D. C. Morse, Phys. Rev. Lett. 108, 238301 (2012).

[39] E. M. Lennon, K. Katsov, and G. H. Fredrickson, Phys. Rev. Lett. 101, 138302 (2008). 